\title{
A qualitative theory of large games with strategic complementarities
}

\author{
Lukasz Balbus ${ }^{1}$. Paweł Dziewulski ${ }^{2}$. \\ Kevin Reffett ${ }^{3}$ - Lukasz Woźny ${ }^{4}$
}

Received: 31 March 2016 / Accepted: 28 August 2017 / Published online: 7 September 2017 (C) The Author(s) 2017. This article is an open access publication

\begin{abstract}
We study the existence and computation of equilibrium in large games with strategic complementarities. Using monotone operators defined on the space of distributions partially ordered with respect to the first-order stochastic dominance, we prove existence of a greatest and least distributional Nash equilibrium. In particular, we obtain our results under a different set of conditions than those in the existing literature. Moreover, we provide computable monotone distributional equilibrium comparative statics with respect to the parameters of the game. Finally, we apply our results to models of social distance, large stopping games, keeping up with the Joneses, as well as a general class of linear non-atomic games.
\end{abstract}

Keywords Large games · Distributional equilibria - Supermodular games · Games with strategic complementarities - Computation of equilibria - Non-aggregative games

\section{JEL Classification C72}

\section{Introduction}

Beginning with the seminal work of Schmeidler (1973) and Mas-Colell (1984), there has been a great deal of work in the economic literature focusing on games with a

$凶$ Łukasz Woźny

lukasz.wozny@sgh.waw.pl

1 Faculty of Mathematics, Computer Sciences and Econometrics, University of Zielona Góra, Zielona Góra, Poland

2 Department of Economics, University of Oxford, Oxford, UK

3 Department of Economics, Arizona State University, Tempe, AZ, USA

4 Department of Quantitative Economics, Warsaw School of Economics, al. Niepodległości 162, 02-554 Warsaw, Poland 
continuum of players. ${ }^{1}$ In a separate, yet related set of papers, researchers have turned their attention to the question of the existence of equilibrium comparative statics in large games with strategic complementarities (henceforth LGSC) between player actions or "traits" [e.g., see Guesnerie and Jara-Moroni (2011) or Acemoglu and Jensen $(2010,2013)]$. The latter strand of work focused primarily on non-atomic, aggregative games, in which payoffs of individual players were affected by an aggregate of actions of other players in the game (or a vector of aggregates). In such games, the questions of equilibrium existence, its computation, and equilibrium comparative statics could be simplified to a great extent. ${ }^{2}$

As we show in this paper, there are classes of large games with a continuum of players in which the payoff of an individual agent depends inherently on the entire distribution of actions and characteristics of other players. Such games cannot be analyzed using the "aggregative games" toolkit. In particular, none of these results in the existing literature that provide positive answers for existence, computation, or comparative statics of equilibria can be applied to such distributional games. Finally, the importance of studying large non-aggregative games with "traits" or diverse personal characteristics has been highlighted, among others, by the recent papers of Khan et al. (2013a,b). In these papers, the authors stress the cardinality of traits as a key factor not only to verify conditions of equilibrium existence, but also to use large games in the study of actual economic problems.

In this paper, we ask a number of questions. First, can the methods used to verify the existence of equilibrium in games with strategic complementarities (with a finite number of players; henceforth GSC) be extended to a general class of games with a continuum of players? If this is possible, can one develop conditions for equilibrium comparative statics results and what will be their nature? Finally, can we develop a theory of computable equilibrium comparative statics for these games?

We begin our analysis in Sect. 2, where we provide new sufficient conditions under which there exists a distributional equilibrium of a LGSC with complete information. In addition, we provide a sharp order-theoretic characterization of the set of equilibria. This characterization plays a central role in developing results on equilibrium comparative statics, as well as when providing an explicit iterative method for computing extremal equilibria. Specifically, by applying constructive results from the literature on order continuous operators, we are able to relax some key continuity conditions on payoffs which are typically required for equilibrium existence in the existing literature that seek to apply standard topological arguments (e.g., continuity in weak topologies).

Moreover, this approach provides constructive methods for computing the extremal equilibria by their successive approximation starting from the extremal elements of the space of all feasible distributions of player actions and "traits". To our knowledge, there are no similar results in the existing literature on computation of extremal equilibria

\footnotetext{
1 For example, see Khan et al. (2013a, b), and the references within.

2 Actually, in many large aggregative games with strategic complementarities, the existence and equilibrium comparative statics questions can be obtained using the standard tools from the theory of game of strategic complementarities with a finite number of players (as in the seminal work of Topkis 1979; Vives 1990; Milgrom and Roberts 1990). However, these tools are not applicable in more general classes of LGSC. See Balbus et al. (2015a) for a discussion.
} 
in LGSC. This result proves to be central in identifying sufficient conditions for the existence of computable equilibrium comparative statics with respect to parameters of a game.

The central point this paper makes is, although the tools used for a study of equilibrium in GSC and LGSC are similar in a very broad methodological sense, the particulars of the methods are significantly different. This dissimilarity arises due to the inherent infinite-dimensional structure of large games. In the framework we develop, joint strategies of players as well as equilibria are defined in terms of probability distributions. Since, in general, sets of probability distributions are not lattices, the methods applied in the analysis for GSC do not apply when seeking to construct and characterize equilibria for LGSC. For example, even though it is possible to provide conditions under which the set of distributional equilibria has greatest and least element, the set of distributional equilibria is, in general, not a complete lattice. ${ }^{3}$ Rather, at most, we are able to show that whenever the best responses of players are functions, the set of distributional equilibria is a chain complete partially ordered set (but not necessarily a complete lattice). ${ }^{4}$

Additionally, the measurability issues induced by the infinite-dimensional specification of LGSC create significant impediments in determining the existence of an equilibrium of the game, let alone characterizing the natures of equilibrium monotone comparative statics. An alternative approach to the measurability issue was recently proposed by Yang and Qi (2013). In this paper, the authors restrict attention to equilibria in strategies that are monotone with respect to traits/characteristics. Their key assumption that the set of traits/characteristics is a chain allows them to apply the results from the existing literature on monotone games. ${ }^{5}$ In general, such specialization is not needed. Rather, we show in this paper for our general specification of a LGSC one does not need to impose significant restrictions on the set of equilibrium strategies, nor the class of games under consideration (aside from measurability). Importantly, in this paper, we also distinguish the need for sufficient complementarities for the existence of a monotone operator on the space of distributional strategies, as opposed to requiring this existence argument be associated with monotone best responses (in names/traits). We show, for a constructive theory of LSGC, we only need a monotone operator; not monotone equilibria. In fact, in Sect. 3 we apply our methods to economic problems in which strategies of players are non-monotone with respect to players characteristics. As a result, both the techniques used and the results obtained in our paper differ in many important dimensions from the environments studied in Yang and Qi (2013) and Bilancini and Boncinelli (2016).

\footnotetext{
${ }^{3}$ A complete lattice is a partially ordered set $(X, \geq)$ such that, for any subset $Y \subseteq X$, the supremum and infimum of $Y$ (with respect to $\geq$ ) belongs to $X$.

4 A partially ordered set (henceforth a poset) $X$ is chain complete (henceforth CPO), if for an arbitrary chain $C \subseteq X$, supremum of $C$ and infimum of $C$ are each in $X$. If this completeness condition holds only for countable chains, we say that $X$ is countably chain complete (henceforth CCPO).

5 As we discuss in a related paper (see Balbus et al. 2015c), the results of Yang and Qi (2013) require stronger conditions on the traits/characteristics space than those imposed in their paper. See a counterexample in Sect. 2 of Balbus et al. (2015c).
} 
The remainder of the paper is organized as follows. In Sect. 2, we provide our main results on the existence of distributional equilibrium, as well as our results on computable equilibrium comparative statics. We also discuss the relationship between our results, and those in the existing literature. In Sect. 3, we show how our results can be applied to Akerlof's social distance model, large optimal stopping games, keeping up with the Joneses, as well as a general class of linear non-atomic supermodular games. Finally, in the "Appendix", we introduce the requisite mathematical terminology used in the paper, as well as some important auxiliary results.

\section{Distributional equilibria in large games}

Let $\Lambda$ be a compact, perfect Hausdorff topological space of player characteristics. ${ }^{6}$ Endow $\Lambda$ with the Borel $\sigma$-field $\mathcal{L}$ and a regular probability measure $\lambda$ vanishing at each singleton. ${ }^{7}$ Let $A \subseteq \mathbb{R}^{m}(m \in \mathbb{N})$ be an action set endowed with the natural product order and Euclidean topology. ${ }^{8}$ By $\tilde{A}: \Lambda \rightarrow 2^{A}$ we denote a correspondence that assigns a sets of feasible actions $\tilde{A}(\alpha) \subseteq A$ to each player $\alpha \in \Lambda$.

By $\mathcal{A}$ we denote a family of Borel subsets on $A$. Let $\mathcal{R}$ denote the set of regular probability measures defined over $\mathcal{L} \otimes \mathcal{A}$, with the marginal distribution on $\Lambda$ equal to $\lambda .{ }^{9}$ Endow $\Lambda \times A$ with a partial order $\geq_{p}$ that satisfies: ${ }^{10}$

$$
\left(\alpha^{\prime}, a^{\prime}\right) \geq_{p}(\alpha, a) \Rightarrow a^{\prime} \geq a .
$$

Observe the partial order $\geq_{p}$ and any action $a \in A$ induce a partial order on $\Lambda$, say $\geq_{p}^{a}$, defined as follows: $\alpha \geq_{p}^{a} \alpha^{\prime}$ if and only if $(\alpha, a) \geq_{p}\left(\alpha^{\prime}, a\right)$. Additionally, assume that, for each $a \in A$ and $\alpha \in \Lambda$, a closed interval of the form $\left\{\alpha^{\prime} \in \Lambda: \alpha^{\prime} \geq_{p}^{a} \alpha\right\}$ belongs to $\mathcal{L}$. This clearly holds if all closed intervals $\left\{\left(\alpha^{\prime}, a^{\prime}\right):\left(\alpha^{\prime}, a^{\prime}\right) \geq_{p}(\alpha, a)\right\}$ belong to $\mathcal{L} \otimes \mathcal{A}$, for all pairs $(\alpha, a)$.

\footnotetext{
6 A topological space is perfect, if all of its elements are accumulation points.

7 Observe that it does not imply that $\lambda$ is a non-atomic measure (see Lemma 12.18 in Aliprantis and Border 2006). Moreover, given that $\Lambda$ is perfect, Theorem 12.21 in Aliprantis and Border (2006) implies that such measure exists. In fact, the assumption that $\Lambda$ is perfect is crucial. For example, let $\Lambda$ be a set of ordinals $\left[0, \omega_{1}\right]$ where $\omega_{1}$ is the first uncountable number. Let $\mathcal{L}$ be the order topology, while measure $\lambda$ vanishes at each singleton. Then, all closed sets not containing $\omega_{1}$ are countable. Hence, whenever we take any neighborhood of $\omega_{1}$, its complement is $\lambda$-null set. Therefore, any neighborhood of $\omega_{1}$ has a full measure, while $\lambda\left(\left\{\omega_{1}\right\}\right)=0$. Each probability measure vanishing at a singleton is not regular. In addition, all successors are isolated points.

8 We assume that the dimension of the action space is finite. See Sun and Zhang (2015) for a discussion on non-atomic games with infinite-dimensional action spaces.

9 The measure is regular, if $\lambda(X)<\infty$, for any compact set $X \in \mathcal{L}$, and if it is both outer regular and tight. Additionally, note that since $\Lambda$ is a compact Hausdorff space, any tight measure is at the same time inner regular. Therefore, any regular measure is both inner and outer regular, hence, normal.

10 Clearly, if $\Lambda$ is an ordered set, this implication is satisfied whenever $\geq p$ is a product order. However, the implication may also be satisfied if the order is such that $\left(\alpha^{\prime}, a^{\prime}\right) \geq p(\alpha, a)$ iff $\left(\alpha=\alpha^{\prime}\right)$ and $\left(a^{\prime} \geq a\right)$. In particular, this allows us to study the case where the set of players has only trivial orders.
} 
Endow $\mathcal{R}$ with the first-order stochastic dominance partial order and denote it by $\succeq_{P} \cdot{ }^{11}$ By Lemma A1 (see the "Appendix"), pair $\left(\mathcal{R}, \succeq_{P}\right)$ constitutes a partially ordered set. $^{12}$ Let

$$
\mathcal{D}:=\{\tau \in \mathcal{R}: \tau(\operatorname{Gr}(\tilde{A}))=1\}
$$

where $\operatorname{Gr}(\tilde{A})$ is the graph of correspondence $\tilde{A}$, denote the set of all feasible distributions. Endow $\mathcal{D}$ with the weak topology and consider a payoff function $r: \Lambda \times A \times \mathcal{D} \rightarrow$ $\mathbb{R}$. We define a game by $\Gamma:=((\Lambda, \mathcal{L}, \lambda), A, \tilde{A}, r) .{ }^{13}$

Definition 1 (Distributional equilibrium) A distributional equilibrium of the game $\Gamma$ is a probability measure $\tau^{*} \in \mathcal{D}$ such that:

$$
\tau^{*}\left(\left\{(\alpha, a): r\left(\alpha, a, \tau^{*}\right) \geq r\left(\alpha, a^{\prime}, \tau^{*}\right), \text { for all } a^{\prime} \in \tilde{A}(\alpha)\right\}\right)=1 .
$$

\subsection{The main result}

Our initial interest is to determine sufficient conditions for the existence of a distributional equilibrium of a LGSC. We impose the following assumptions:

\section{Assumption 1 Assume that}

(i) mapping $\tilde{A}$ is weakly $\mathcal{L}$-measurable correspondence taking values that are complete sublattices of $\mathbb{R}^{m 14}$;

(ii) for $\lambda$-a.e. player $\alpha \in \Lambda$, function $r$ is quasisupermodular over $A$ and has single crossing differences in $(a, \tau)$ on $A \times \mathcal{D} ;{ }^{15}$

11 That is, we have $\tau^{\prime} \succeq_{P} \tau$ if and only if $\int f(\alpha, a) \mathrm{d} \tau^{\prime}(\alpha, a) \geq \int f(\alpha, a) \mathrm{d} \tau(\alpha, a)$, for any increasing (with respect to $\geq_{p}$ ), bounded, and measurable function $f: \Lambda \times A \rightarrow \mathbb{R}_{+}$.

12 The set of all isotone with respect to $\geq_{p}$ and measurable functions $f: \Lambda \times A \rightarrow \mathbb{R}$ separates the points in $\Lambda \times A$. To see this, take any $\left(\alpha_{1}, a_{1}\right)$ and $\left(\alpha_{2}, a_{2}\right)$. Assume $a_{1}$ is no greater then $a_{2}$. Define $f(\alpha, a)=\chi_{\left\{a^{\prime} \in A: a \geq a_{2}\right\}}(a)$. Clearly, function $f$ is isotone with respect to $\geq_{p}$. Finally, it is $\mathcal{L} \otimes \mathcal{A}$ measurable, as $0=f\left(\alpha_{1}, a_{1}\right) \neq f\left(\alpha_{2}, a_{2}\right)=1$. Let $a_{1}=a_{2}=a$ and $\alpha_{1} \ngtr a \alpha_{2}$. Then, by our assumption on $\geq p$, all points of the form $\left(\alpha_{1}, a\right)$ and $\left(\alpha_{2}, a\right)$ may be separated by the indicator of $\left\{\alpha^{\prime}: \alpha^{\prime} \geq p \alpha_{2}\right\}$.

13 Unlike Mas-Colell (1984), who characterized players by their payoff functions only, in our specification the space of characteristics is equivalent to the measure space $(\Lambda, \mathcal{L}, \lambda)$. Nevertheless, once we define $r(\alpha, a, \tau):=\alpha(a, \tau)$, where $\Lambda:=\{\alpha:(a, \tau) \rightarrow \alpha(a, \tau)$ is a continuous function $\}$, our framework embeds the one proposed by Mas-Colell (1984). Alternatively, we can interpret $\alpha$ as a fixed trait, just like we do in Sect. 3. See also Khan et al. (2013a, b), who analyze games with traits.

14 We use symbols $\bigvee$ and $\bigwedge$ to denote the sup and inf of an underlying set, induced by the corresponding partial order. We refer to these selections as the "extremal" selections. A sublattice $Y$ of $X$ is complete whenever, for any subset $C \subseteq Y, \bigvee C:=\sup (C)$ and $\bigwedge C:=\inf (C)$ belong to $Y$, where the sup/inf operators $\bigvee / \bigwedge$ are defined with respect to the induced order of $X$. Thus, the extremal elements of a complete sublattice are greatest and least elements. Moreover, since $\tilde{A}(\alpha) \subseteq \mathbb{R}^{m}$ is a complete sublattice, correspondence $\tilde{A}$ is compact-valued (e.g., see Theorem 2.3.1 in Topkis 1998).

15 Suppose that $\left(X, \geq_{X}\right)$ is a lattice and $\left(Y, \geq_{Y}\right)$ is a partially ordered set. We say that function $f: X \rightarrow \mathbb{R}$ is quasisupermodular over $X$ if, for any $x, x^{\prime}$ in $X, f(x) \geq f\left(x \wedge x^{\prime}\right)$ implies $f\left(x \vee x^{\prime}\right) \geq f\left(x^{\prime}\right)$, and $f(x)>f\left(x \wedge x^{\prime}\right)$ implies $f\left(x \vee x^{\prime}\right)>f\left(x^{\prime}\right)$. Moreover, function $g: X \times Y \rightarrow \mathbb{R}$ has single crossing differences in $(x, y)$ on $X \times Y$ whenever, for any $x^{\prime} \geq_{X} x$ in $X$ and $y^{\prime} \geq_{Y} y$ in $Y, g\left(x^{\prime}, y\right) \geq g(x, y)$ implies $g\left(x^{\prime}, y^{\prime}\right) \geq g\left(x, y^{\prime}\right)$, and $g\left(x^{\prime}, y\right)>g(x, y)$ implies $g\left(x^{\prime}, y^{\prime}\right)>g\left(x, y^{\prime}\right)$. 
(iii) for any $\tau \in \mathcal{D}$, function $(\alpha, a) \rightarrow r(\alpha, a, \tau)$ is Carathéodory. ${ }^{16}$

In order to proceed with our result on the existence of a distributional equilibrium, we define the best response correspondence $m: \Lambda \times \mathcal{D} \rightarrow 2^{A}$ by:

$$
m(\alpha, \tau):=\underset{a \in \tilde{A}(\alpha)}{\operatorname{argmax}} r(\alpha, a, \tau)
$$

with its greatest selection $\bar{m}: \Lambda \times \mathcal{D} \rightarrow A$, where $\bar{m}(\alpha, \tau):=\bigvee m(\alpha, \tau)$. Similarly, denote the least selection $\underline{m}: \Lambda \times \mathcal{D} \rightarrow A$, where $\underline{m}(\alpha, \tau):=\bigwedge m(\alpha, \tau)$. Define the upper distributional operator $\bar{T}: \mathcal{D} \rightarrow \mathcal{D}$ by:

$$
(\bar{T} \tau)(G):=\lambda(\{\alpha \in \Lambda:(\alpha, \bar{m}(\alpha, \tau)) \in G\}) \text {, for any } G \in \mathcal{L} \otimes \mathcal{A} .
$$

Similarly, using the least best reply $\underline{m}(\alpha, \tau)$, we can define the lower distributional operator $\underline{T}$.

First, we characterize the monotonicity properties of the two operators.

Lemma 1 Operators $\bar{T}$ and $\underline{T}$ are well defined and $\succeq_{P}$-increasing.

Proof We prove the result for operator $\bar{T}$. Using an analogous argument, we can show that the lemma is also true for $\underline{T}$.

Claim 1: Operator $\bar{T}$ is well defined. Since $r$ is continuous and quasisupermodular on $A$, by Theorem 4 in Milgrom and Shannon (1994), $m(\alpha, \tau)$ is a complete sublattice of $A$, for any $\alpha \in \Lambda$ and $\tau \in \mathcal{D}$. In particular, set $m(\alpha, \tau)$ is non-empty and $\bar{m}(\alpha, \tau):=$ $\bigvee m(\alpha, \tau)$ is well defined, for any $\alpha \in \Lambda$ and $\tau \in \mathcal{D}$.

Next, we show that correspondence $m$ has an increasing measurable selector. Given that function $r$ is Carathéodory, for all $\tau \in \mathcal{D}$, and correspondence $\tilde{A}$ is weakly measurable, by Theorem 18.19 in Aliprantis and Border (2006), correspondence $m$ is $\mathcal{L}$-measurable, for any $\tau \in \mathcal{D}$. As $m(\cdot, \tau)$ maps a measurable space into a metrizable space, it is also weakly measurable (see Theorem 18.2 in Aliprantis and Border 2006).

Let $\pi_{i}: \mathbb{R}^{m} \rightarrow \mathbb{R}$, be a projection defined by $\pi_{i}\left(x_{1}, \ldots, x_{i}, \ldots, x_{m}\right):=x_{i}$, for any $\left(x_{1}, \ldots, x_{m}\right) \in \mathbb{R}^{m}$. Note that, $\bar{m}(\alpha, \tau)=\left(\bar{a}_{1}, \ldots, \bar{a}_{m}\right)$, where we denote $\bar{a}_{i}:=$ $\max _{a \in m(\alpha, \tau)} \pi_{i}(a)$. Since the projection $\pi_{i}$ is a continuous function, for any $i=$ $1, \ldots, m$, while correspondence $m(\cdot, \tau)$ is weakly measurable, by Theorem 18.19 in Aliprantis and Border (2006), function $\bar{m}(\alpha, \tau)$ is $\lambda$-measurable. Therefore, by Himmelberg's Theorem (e.g., Lemma 18.4 in Aliprantis and Border 2006), function $\bar{m}(\cdot, \tau)$ is $\mathcal{L}$-measurable, for any $\tau \in \mathcal{D}$.

In order to complete the first part of the proof, we need to show for any $\tau \in \mathcal{D}$, the measure $(\bar{T} \tau)$ belongs to $\mathcal{D}$. Clearly, $(\bar{T} \tau)(\operatorname{Gr}(\tilde{A}))=1$. Hence, it suffices to show that $(\bar{T} \tau)$ is normal and regular. To see this, recall that as the marginal distribution of $(\bar{T} \tau)$ over $\Lambda$ is equal to $\lambda$, it is regular. Moreover, since the marginal distribution of $(\bar{T} \tau)$ over metrizable $A$ is a bounded Borel measure, by Theorem 12.5 in Aliprantis and Border (2006), it is regular. Therefore, by Lemma A2 (see the "Appendix"), measure $(\bar{T} \tau)$ is regular.

$\overline{16}$ That is, the function $(\alpha, a) \rightarrow r(\alpha, a, \tau)$ is continuous on $A$ and $\mathcal{L}$-measurable. 
Claim 2: Operator $\bar{T}$ is $\succeq_{P}$-isotone. Since, for $\lambda$-a.e. $\alpha \in \Lambda$, function $r$ has single crossing differences in $(a, \tau)$ on $A \times \mathcal{D}$, by Theorem 4 in Milgrom and Shannon (1994), correspondence $m$ is isotone in the strong set order on $\mathcal{D}$, for $\lambda$-a.e. $\alpha \in \Lambda$. In particular, this implies that function $\bar{m}$ increases on $\mathcal{D}$, for $\lambda$-a.e. $\alpha \in \Lambda$. As a result, for $\lambda$-a.e. $\alpha \in \Lambda$, we have $\bar{m}\left(\alpha, \tau^{\prime}\right) \geq \bar{m}(\alpha, \tau)$, whenever $\tau^{\prime} \succeq_{P} \tau$. We show that $\left(\bar{T} \tau^{\prime}\right) \succeq_{P}(\bar{T} \tau)$. For any increasing and measurable function $f: \Lambda \times A \rightarrow \mathbb{R}$,

$$
\begin{aligned}
\int_{\Lambda \times A} f(\alpha, a) \mathrm{d}\left(\bar{T} \tau^{\prime}\right)(\alpha, a) & =\int_{\Lambda} f\left(\alpha, \bar{m}\left(\alpha, \tau^{\prime}\right)\right) \mathrm{d} \lambda(\alpha) \\
& \geq \int_{\Lambda} f(\alpha, \bar{m}(\alpha, \tau)) \mathrm{d} \lambda(\alpha)=\int_{\Lambda \times A} f(\alpha, a) \mathrm{d}(\bar{T} \tau)(\alpha, a),
\end{aligned}
$$

where the first and last equality follows from the definition of $\bar{T}$, while the inequality is implied by monotonicity of $f$ and $\bar{m}(\alpha, \cdot)$.

Our next result characterizes the order structure of the partially ordered $\operatorname{set}\left(\mathcal{D}, \succeq_{P}\right)$. The next lemma follows from Proposition A1 (see the "Appendix").

Lemma $2\left(\mathcal{D}, \succeq_{P}\right)$ is a chain complete poset.

With these two lemmas in place, we are ready to state the main theorem of the paper. Let $\bar{\delta}$ and $\underline{\delta}$ denote a greatest and a least elements of set $\mathcal{D}$.

\section{Theorem 1 Under Assumption 1}

(i) there exists a greatest and a least distributional equilibrium of $\Gamma$;

(ii) if $\underline{T}=\bar{T}$ (i.e., if the best response correspondence is a function), then the set of distributional equilibria is a chain complete poset. ${ }^{17}$

Denote greatest and least equilibrium of $\Gamma$ by $\bar{\tau}^{*}$ and $\tau^{*}$, respectively. If for any countable chain $\left\{\tau_{n}\right\} \subseteq \mathcal{D}$ such that $\tau_{n} \rightarrow \tau$, we have $r\left(\alpha, a, \tau_{n}\right) \rightarrow r(\alpha, a, \tau)$ :

(iii) $\bar{\tau}^{*}=\lim _{n \rightarrow \infty} \bar{T}^{n}(\bar{\delta})$ and $\underline{\tau}^{*}=\lim _{n \rightarrow \infty} \underline{T}^{n}(\underline{\delta})$.

Proof We present the argument via a number of claims.

Claim 1: The greatest fixed point of $\bar{T}$ is a distributional equilibrium of $\Gamma$. By Lemma 2 , the pair $\left(\mathcal{D}, \succeq_{P}\right)$ is a chain complete poset. By Lemma $1, \bar{T}$ is a welldefined, $\succeq_{P}$-isotone operator that maps $\mathcal{D}$ into itself. Therefore, by Theorem A1 (see the "Appendix") we conclude that the set of fixed points of operator $\bar{T}$ is non-empty and has a greatest element, denoted by $\bar{\tau}^{*}$. Clearly, $\bar{\tau}^{*} \in \mathcal{D}$. Moreover, we have:

$$
\bar{\tau}^{*}\left(\left\{(\alpha, a): a \in m\left(\alpha, \tau^{*}\right)\right\}\right) \geq \bar{\tau}^{*}\left(\left\{(\alpha, a): a=\bar{m}\left(\alpha, \tau^{*}\right)\right\}\right)=1,
$$

which implies $\bar{\tau}^{*}$ is a distributional equilibrium of $\Gamma$. By a dual argument, we can show that the least fixed point of operator $\underline{T}$, denoted by $\underline{\tau}^{*}$, is a distributional equilibrium of $\Gamma$.

\footnotetext{
17 Conditions under which the best response correspondence is a function are well known. For example, whenever correspondence $\tilde{A}$ is convex-valued and the payoff function $r$ is strictly quasi-concave on $A$.
} 
Claim 2: $\bar{\tau}^{*}$ is a greatest distributional equilibrium of $\Gamma$. Let $\tau$ be any equilibrium of the game. By the definition of distributional equilibria, we have:

$$
1=\tau(\{(\alpha, a): a \in m(\alpha, \tau)\}) \leq \tau(\{(\alpha, a): a \leq \bar{m}(\alpha, \tau)\}) .
$$

Therefore, $\tau$ is concentrated over the set $E:=\{(\alpha, a): a \leq \bar{m}(\alpha, \tau)\}$. Take any increasing function $f: \Lambda \times A \rightarrow \mathbb{R}$. Then,

$$
\begin{aligned}
\int_{\Lambda \times A} f(\alpha, a) \mathrm{d} \tau(\alpha, a) & =\int_{E} f(\alpha, a) \mathrm{d} \tau(\alpha, a) \\
& \leq \int_{\Lambda} f(\alpha, \bar{m}(\alpha, \tau)) \mathrm{d} \lambda(\alpha)=\int_{\Lambda \times A} f(\alpha, a) \mathrm{d}(\bar{T} \tau)(\alpha, a),
\end{aligned}
$$

where the inequality follows from the definition of $E$ and the final equation is implied by the definition of $\bar{T}$. Therefore, $(\bar{T} \tau) \succeq_{P} \tau$. Since $\bar{T}$ is $\succeq_{P}$-isotone, by Theorem A2 (see the "Appendix"), we have that $\bar{\tau}^{*} \succeq_{P} \tau$. Hence, $\bar{\tau}^{*}$ is a greatest distributional equilibrium of $\Gamma$. Again, by a dual argument, we can show that $\underline{\tau}^{*}$ is the least distributional equilibrium of $\Gamma$.

Observe Claims 1 and 2 prove statement (i) of Theorem 1. Statement (ii) is directly implied by Theorem A1 (see the "Appendix"). Since the set of fixed points of operator $T=\bar{T}=\underline{T}$ is a chain complete poset, the set of distributional equilibria of $\Gamma$ is also chain complete.

In the remainder of the proof we assume that, for any countable chain $\left\{\tau_{n}\right\} \subseteq \mathcal{D}$ such that $\tau_{n} \rightarrow \tau$, we have $r\left(\alpha, a, \tau_{n}, s\right) \rightarrow r(\alpha, a, \tau, s)$.

Claim 3: Operator $\bar{T}$ is monotonically inf-preserving. Let $\left\{\tau_{n}\right\} \subseteq \mathcal{D}$ be a monotonically decreasing sequence with infimum $\tau$. By Lemma 2, we have $\tau \in \mathcal{D}$. Moreover,

$$
r\left(\alpha, \bar{m}\left(\alpha, \tau_{n}\right), \tau_{n}\right) \geq r\left(\alpha, a, \tau_{n}\right)
$$

Since $\bar{m}\left(\alpha, \tau_{n}\right)$ belongs to $\tilde{A}(\alpha)$ which is compact, the limit of this sequence, denoted by $\bar{m}^{*}$, exists and belongs to $\tilde{A}(\alpha)$. By continuity of $r$, we have $r\left(\alpha, \bar{m}^{*}, \tau\right) \geq$ $r(\alpha, a, \tau)$, for all $a \in \tilde{A}(\alpha)$. Therefore, $\bar{m}^{*} \in m(\alpha, \tau)$ and

$$
\bar{m}^{*} \leq \bar{m}(\alpha, \tau)
$$

As $\tau_{n} \succeq_{P} \tau$, for all $n$, by monotonicity of $\bar{m}$, we have $\bar{m}(\alpha, \tau) \leq \bar{m}\left(\alpha, \tau_{n}\right)$ and

$$
\bar{m}(\alpha, \tau) \leq \lim _{n \rightarrow \infty} \bar{m}\left(\alpha, \tau_{n}\right)=\bar{m}^{*}
$$

Combining (1) and (2) implies $\bar{m}(\alpha, \tau)=\lim _{n \rightarrow \infty} \bar{m}\left(\alpha, \tau_{n}\right)$. Therefore, $\bar{T}$ is monotonically inf-preserving. By a dual argument, we show that $\underline{T}$ is monotonically sup-preserving.

Claim 4: $\bar{\tau}^{*}=\lim _{n \rightarrow \infty} \bar{T}^{n}(\bar{\delta})$. By Claim 3, operator $\bar{T}$ is monotonically infpreserving. Moreover, it is also $\succeq_{P}$-isotone. Therefore, by Theorem A2 we conclude that $\bar{T}^{n}(\bar{\delta}) \rightarrow \bar{\tau}^{*}$. Analogously, by a dual argument, we can show that $\underline{\tau}^{*}=$ $\lim _{n \rightarrow \infty} \underline{T}^{n}(\underline{\delta})$. 
We next turn to the question of monotone comparative statics of the set of distributional equilibria of a LGSC. In particular, our concern is not only to prove the existence of distributional comparative statics, but also to provide sufficient conditions that would allow for their computation. Given our generalization of Markowsky's Theorem (see Theorem A4 in the "Appendix"), we are able to characterize the order structure of the set of fixed points of sup- and inf-preserving maps defined over countably chain complete posets.

Before stating our next result, we need to introduce some additional notation. Consider a parameterized version of our large game

$$
\Gamma(s):=((\Lambda, \mathcal{A}, \lambda), A(\cdot, s), \tilde{A}(\cdot, s), r(\cdot, s)),
$$

for each $s \in S$, where the space of parameters $\left(S, \geq_{S}\right)$ forms a partially ordered set. We impose the following assumptions of $\Gamma(s)$.

\section{Assumption 2 For any $s \in S$, suppose that}

(i) correspondence $\tilde{A}(\cdot, s)$ and function $r(\cdot, s)$ obey Assumption 1 ;

(ii) for all $\alpha \in \Lambda$, mapping $s \rightrightarrows \tilde{A}(\alpha, s)$ increases in the strong set order; $;^{18}$

(iii) for $\lambda$-a.e. $\alpha$, function $r$ has single crossing differences in $(a, s)$ on $A \times S$;

(iv) for any countable chain $\left\{\tau_{n}\right\} \subseteq \mathcal{D}, \tau_{n} \rightarrow \tau$ implies $r\left(\alpha, a, \tau_{n}, s\right) \rightarrow r(\alpha, a, \tau, s)$.

Under the above assumption, we can prove the following equilibrium monotone comparative statics result.

Corollary 1 Under Assumption 2, for any $s \in S$, there exist a greatest and a least distributional equilibrium of $\Gamma(s)$, denoted by $\bar{\tau}^{*}(s)$ and $\underline{\tau}^{*}(s)$ respectively. Moreover, functions $s \rightarrow \bar{\tau}^{*}(s)$ and $s \rightarrow \underline{\tau}^{*}(s)$ are increasing.

The above corollary follows from Theorems 1 and A4 (see the "Appendix").

\subsection{Remarks and discussion}

In this subsection, we discuss three issues. First, we compare our results to the related work in the existing literature concerning distributional equilibria in large games. Then, we discuss how the structure of the set of equilibria in LGSC differs from the one in GSC (with a finite number of players). Finally, we relate our results to the literature on monotone equilibrium comparative statics in games with a continuum of players.

Theorem 1 establishes the existence of distributional equilibria under a different set of assumptions than in Mas-Colell (1984) or in other related papers in the literature that followed. In particular, we do not require for the payoff function $r$ to be weakly continuous with respect to distributions $\tau$ on $\mathcal{D}$. Instead, we endow sets $\Lambda \times A$ and $\mathcal{D}$

\footnotetext{
18 Suppose that $\left(X, \geq_{X}\right)$ is a poset, while $\left(Y, \geq_{Y}\right)$ is a lattice. Correspondence $F: X \rightarrow 2^{Y}$ is ascending in the strong set order if, for any $x^{\prime} \geq_{X} x$ in $X$ and $y \in F(x), y^{\prime} \in F\left(x^{\prime}\right)$, we have $y \wedge y^{\prime} \in F(x)$ and $y \vee y^{\prime} \in F\left(x^{\prime}\right)$. Clearly, the above definition implies that correspondence $F$ is sublattice-valued.
} 
with a partially ordered structure, impose the quasisupermodularity and single crossing differences conditions on the payoff function, and require the set of feasible actions of players is a complete sublattice of $\mathbb{R}^{m}$. In this sense, our approach allows to analyze games with discontinuous payoffs. ${ }^{19}$ See also Rath (1996), Carmona and Podczek (2014) or Prokopovych and Yannelis (2017).

Second, our main theorem establishes the existence of extremal distributional equilibria which, aside from generalizing some of the results for GSC, allows us to develop the order-theoretic characterization of distributional equilibria. Furthermore, under stronger continuity conditions on payoffs, it provides a method of computing the equilibrium comparative statics (as well as extremal distributional equilibria at any fixed parameter). Additionally, given Theorem 1(ii), in a special case we provide an ordertheoretic characterization of the entire set of distributional equilibria. Therefore, this result highlights some aspects of the existence theorems obtained for GSC via various versions of Tarski's Theorem (see Veinott 1992; Zhou 1994).

However, it is worth mentioning that, unlike in GSC, we do not expect the set of distributional equilibria in LGSC to be a complete lattice. In fact, given Theorem 1(ii), we can at most expect that the set is a chain complete poset. The reason for this weaker characterization of equilibrium is very simple. In general, although the action set $A$ is a complete lattice, the set of distributions over $A$ is not. ${ }^{20}$ As a result, instead of applying the result of Tarski (1955), we must appeal to an alternative result by Markowsky (1976). ${ }^{21}$

Our approach suggests a direct method for computing particular distributional equilibria of a game as well as their monotone comparative statics (compare with Chapter 4.3 in Topkis 1998). Under sufficient order-continuity conditions imposed on payoffs, in order to compute the extremal distributional equilibria, one needs to calculate the order-limit of a sequence generated by the upper and the lower distributional operator $\bar{T}$ and $\underline{T}$. The sequences are generated by iterating downward (respectively upwards) from greatest (respectively least) element of the chain complete poset $\left(\mathcal{D}, \succeq_{P}\right)$. Given that operators $\bar{T}$ and $\underline{T}$ are monotonically inf- and sup-preserving, the order-limits of our iterations are attainable in a countable number of steps. Hence, they are computable. $^{22}$ Nevertheless, in order to obtain the computability result, we require that payoffs are weakly continuous on the space of distributions $\mathcal{D}$. Unlike in the original result by Mas-Colell (1984), the weak continuity assumption in not critical for existence of an equilibrium; rather, we only need this condition for the computation/successive approximation of the extremal equilibria and their computable comparative statics.

\footnotetext{
19 For example, our methods allow to analyze large games of Bertrand competition in which the demand function is discontinuous due to product substitutability. Observe that this class of games cannot be analyzed under the assumptions imposed by, e.g., Rath (1996).

${ }^{20}$ For example, consider set of distributions on $A \subseteq \mathbb{R}^{m}, m \geq 2$, ordered by first-order stochastic dominance ordering. In this case, it is well known that the space of probability measures on $A$ is not a lattice (see Kamae et al. 1977); rather, it is only a CPO.

21 It is worth noting that Theorem 11 of Markowsky (1976) characterizes chain complete posets using their fixed-point property relative to increasing mappings. In this sense, Markowsky (1976) provides a converse to Theorem A1, just as Davis (1955) provides a converse to Tarski's Theorem.

22 See the "Appendix" for a discussion of monotonically sup- and inf-preserving mappings.
} 
Finally, the only result concerning the existence of equilibrium comparative statics for large games, of which we are aware, is presented by Acemoglu and Jensen (2010). Their approach to equilibrium comparative statics is very similar to ours, as they impose conditions guaranteeing that the joint best response mapping has increasing selections with respect to the parameters of the game (see Definition 3 in their paper). However, there are differences. First of all, as they concentrate on aggregative games, where players best respond to the average/mean action of other players, the class of games in which they obtain the result is quite different (and more restrictive) than ours. In particular, our framework includes their class of games, but also allows for more general specifications of large games. Second, in case of a single dimensional action space $A$, Acemoglu and Jensen (2010) manage to show the comparative statics of the extremal (aggregative) equilibria without the single crossing property between player actions and aggregates. This constitutes an extension of our results in the case of one dimensional action spaces. However, once the space of actions is multidimensional, Acemoglu and Jensen (2010) require that payoffs have increasing differences in the action of a player and the aggregate, which is stronger than the ordinal notion of single crossing differences that we use. Finally, in order to show existence of an aggregate equilibrium Acemoglu and Jensen (2010) use the topological fixed-point theorem of Kakutani. This makes the issues of equilibrium comparative statics and computability of equilibrium difficult to address. On the other hand, our use of order-theoretical fixed-point results allows to address both issues directly.

We conclude this subsection with some remarks on generality of our results.

Remark 1 In his original paper, Mas-Colell (1984) focused on anonymous games in which the payoff function depended on the marginal distribution over the action space. In particular, the characteristics of agents were not taken into consideration by the players. Hence, the term anonymous. In other words, the reward function took the form of $r(\alpha, \tau):=\tilde{r}\left(\alpha, \tau_{A}\right)$, for some function $\tilde{r}$, where $\tau_{A}$ is the marginal distribution of $\tau$ over the set of actions $A$. It bears mentioning that our framework embeds anonymous games as a special case. Clearly, using a simple transformation $\tau_{A}(\cdot)=\tau(\Lambda \times \cdot)$, we can always construct payoffs that depend on $\tau_{A}$, rather than $\tau$.

Remark 2 After a careful read of the proof of Theorem 1, it is easy to see that the theorem consists of two separate results. The first one states that the greatest fixed point of operator $\bar{T}$ constitutes the greatest distributional equilibrium of $\Gamma$. The second one argues that the least fixed point of operator $\underline{T}$ is the least equilibrium of the game. As long as the two operators are well defined, the two results hold simultaneously. However, if only one of them exists, it is still possible to show that there is either the greatest or the least equilibrium of the game, using the same argument as in the proof of Theorem 1. In particular, this allows us to analyze the class of superextremal games, introduced by Shannon (1990), LiCalzi and Veinott (1992), and Veinott (1992), in the large framework. That is, whenever we allow for the payoff function to exhibit a weaker form of complementarity then quasisupermodularity/lattice superextremal and single crossing property/interval-crossing differences. For example, in LiCalzi and Veinott (1992), the notion of a join (respectively, meet) superextremal and join (meet) upcrossing differences is introduced. In this case, best replies in our games 
for $\tilde{A}$ will be join (meet) sublattice-valued (rather than sublattice-valued in the lattice superextremal/quasisupermodular case with interval-crossing differences/single crossing property). In this case of weaker forms of complementary, appealing to the work of LiCalzi and Veinott (1992), we can guarantee that that the upper (the lower) distributional operator exists. Hence, there is greatest (least) equilibrium of the game. Moreover, the monotone comparative statics of the equilibrium, established in Corollary 1 , also apply. ${ }^{23}$

Remark 3 In Sect. 3 that follows, we concentrate on applications which are inherently infinite-dimensional. That is, we can discuss examples in which players take into account the entire distribution $\tau$ in order to evaluate their payoffs. Nevertheless, there are numerous applications in which agents interact with each other via an aggregate (e.g., see Acemoglu and Jensen 2010; Guesnerie and Jara-Moroni 2011). The results presented in this paper are still applicable to this subclass of games. However, they can be strengthened in this particular framework. ${ }^{24}$ Suppose that the payoff function $r(\alpha, a, g)$ depends on an aggregate value $g$, of players characteristics and actions. Suppose that $g$ is an element of some complete lattice $G$. Moreover, let $g$ be determined by function $h: \mathcal{D} \rightarrow G$, which is increasing on $\mathcal{D} .{ }^{25}$ Define an operator $\bar{\Psi}: G \rightarrow G$ by $\bar{\Psi}(g):=h(\bar{T} g)$, where

$$
(\bar{T} g)(H)=\lambda(\{\alpha:(\alpha, \bar{m}(\alpha, g)) \in H\}), \text { for some } H \in \mathcal{L} \otimes \mathcal{A},
$$

while $\bar{m}(\alpha, g)=\bigvee \operatorname{argmax}_{a \in \tilde{A}(\alpha)} r(\alpha, a, g)$. Whenever Assumption 1 is satisfied with $r$ having single crossing differences in $(a, g)$ on $A \times G$, it is easy to show that operator $\bar{\Psi}$ is well defined and isotone. Since $G$ is a complete lattice, we can apply Tarski's Theorem to show that the operator has a greatest fixed point $\bar{g}^{*}$. This implies that there is a greatest distributional equilibrium of the game $\left(\bar{T} \bar{g}^{*}\right)$. Similarly, we may prove the existence of a least equilibrium.

Remark 4 Following the tradition of Schmeidler (1973), it is possible to define an equilibrium of a LGSC in terms of strategy profiles, rather than distributions. In this framework, a strategy profile is a function $f: \Lambda \rightarrow A$ that assigns an action $f(\alpha) \in$ $\tilde{A}(\alpha)$ to agent $\alpha \in \Lambda$. Moreover, we find it desirable to restrict our attention to $\mathcal{L}$ measurable functions.

To see how could we adapt our techniques to the above concept of equilibrium, define a set of $\mathcal{L}$-measurable functions $f: \Lambda \rightarrow A$ by $\mathcal{F}$. Endow the space with the pointwise order. ${ }^{26}$ Therefore, the payoff function is $r: \Lambda \times A \times \mathcal{F} \rightarrow \mathbb{R}$.

\footnotetext{
23 In the "superextremal" variant of the superextremal class of payoffs (with upcrossing differences), LiCalzi and Veinott (1992), Veinott (1992) prove the existence of monotone selections in best reply maps; an open question is if they are measurable. Our results do not generalize directly to this case.

24 We thank one anonymous referee of this journal for recommending us this application.

25 Let $G \subset \mathbb{R}^{n}$. One example of an aggregator may be $h=\left(h_{1}, \ldots, h_{n}\right)$, where $h_{i}: \mathcal{D} \rightarrow \mathbb{R}$ is defined by $h_{i}(\tau):=\int_{\Lambda \times A} f_{i}(\alpha, a) \mathrm{d} \tau(\alpha, a)$, for some increasing $f_{i}: \Lambda \times A \rightarrow \mathbb{R}, i=1, \ldots, n$.

26 That is, we say that function $f^{\prime}$ dominates $f$ in the pointwise order if $f^{\prime}(\alpha) \geq f(\alpha)$, for all $\alpha \in \Lambda$. Note, this is a different ordering, than the one discussed in our main result.
} 
Under similar conditions to those stated in Assumption 1, it is possible to provide an analogous characterization of the set of equilibria, including the existence of a greatest and a least elements of the equilibrium set. However, the method of proving the result is different. In particular, we are forced to use a different fixed-point result in our argument, as the Markowsky's Theorem is no longer applicable.

The reason we need to refer to a different argument is implied by the fact that, unlike the space of distributions, the set of bounded Borel-measurable functions endowed with the pointwise order is not a chain complete poset. In fact, the set is a $\sigma$-complete lattice, which means that only countable subsets of $\mathcal{F}$ (see Example 2.1 in Heikkilä and Reffett 2006).

Therefore, the set is a countable chain complete. Clearly, this follows from the very definition of measurability, which need not be preserved under uncountable operations. The lack of the strong form of completeness of the joint strategy set forces us to use the Tarski-Kantorovich theorem (see Theorem A2 in the "Appendix") in order to prove the existence of an equilibrium in the sense of Schmeidler (1973). This, on the other hand, requires additional order-continuity assumptions on the payoff function. ${ }^{27}$ Recall in case of distributional equilibria, the continuity conditions were only required for the approximation of the extremal equilibria and the monotone comparative statics, but not for equilibrium existence. Nevertheless, our order-continuity assumptions are weaker than weak continuity assumed in the existing literature (e.g., see Khan 1986). ${ }^{28}$ Moreover, our methods allow for the iterative approximation of the extremal equilibria, and their monotone comparative statics.

\section{Applications}

In this section, we present several economic applications of the methods. In each example, our emphasis is on non-aggregative large games.

\subsection{Social distance model}

To motivate the results in our paper, we begin by considering distributional equilibria in a version of the social distance model described originally by Akerlof (1997). The model studies the distribution of social ranks/statuses over a large number of heterogeneous individuals. Consider a continuum of agents distributed over a compact interval $X \subset \mathbb{R}$, where a location of an individual agent is denoted by $x \in X$. Let $Y \subset \mathbb{R}$ be the set of all possible positions in the society (e.g., social statuses/ranks), where $Y$ is compact and convex. Each individual is characterized by an identity $y \in Y$, which determines the social status/rank to which the agent aspires. We shall refer to the location-identity pair $(x, y)$ as the characteristic of an agent.

\footnotetext{
27 Formally, we require that the payoff function preserves the limits of monotone sequences in $\mathcal{F}$. This guarantees that the extremal best response selections are monotonically inf- and sup-preserving-a feature required for the application of Tarski-Kantorovich theorem.

28 Since order-continuity has to be verified only with respect to all sub-chains of the original set, it is easy to provide an example of payoff functionals that are order-continuous, but not weakly continuous.
} 
Suppose that the distribution of characteristics across the population is determined by a probability measure $\lambda$, defined over the Borel-field of $X \times Y$. We assume that every agent knows his own characteristic $(x, y)$, as well as the distribution of characteristics across the population. In this game, we study how agents determine their optimal individual choice of social status $a \in Y$, given their location $x \in X$ and identity $y \in Y$.

In this model, the payoff of an individual is determined as follows. First of all, every agent aims to attain a status/rank that is in proximity to his true identity $y$. Therefore, the agent will suffer a penalty, whenever his social status $a$ does not match to his true identity $y$. Moreover, the further away the actual status is from the true identity, the more disutility the agent receives.

Second of all, the individual payoff is affected by interactions with other agents in the game. Assume the players meet at random. Whenever an agent meets another player, he suffers a disutility if his social status $a$ differs from the social status $a^{\prime}$ of the other individual. The disutility increases the greater is the distance between the two statuses. This incorporates a form of peer pressure or conformism to the game.

Let $u, v: \mathbb{R} \rightarrow \mathbb{R}$ be continuous, decreasing functions. In addition, assume that $v$ is concave. Consider an agent characterized by $(x, y)$ who chooses a status $a \in Y$. Whenever the agent meets another individual with a social status $a^{\prime} \in Y$, his utility is given by:

$$
u(|a-y|)+v\left(\left|a-a^{\prime}\right|\right) .
$$

As both functions $u$ and $v$ are decreasing, the objective of every player is to choose an action as close as possible to their true identity $y$ and the identity $a^{\prime}$ of the other player. Moreover, given concavity of function $v$, the further away is the status of the agent from the social rank of the other player, the steeper are the changes in the disutility.

In order to make our notation compact, denote $\Lambda:=X \times Y$, with a typical element $\alpha=(x, y)$. Suppose that the frequency of interactions of the agent with other individuals is governed by a probability measure $\mu_{\alpha}$, defined over the product Borel-field of $\Lambda$. Therefore, for any set $U=U_{x} \times U_{y}$, where $U_{x} \subset X$ and $U_{y} \subset Y$, value $\mu_{\alpha}(U)$ is the probability of encountering an agent with a characteristic $\left(x^{\prime}, y^{\prime}\right) \in U_{x} \times U_{y}$. We assume that the measure $\mu_{\alpha}$ depends on $\alpha=(x, y)$, as both the location $x$ of the agent and his aspirations $y$ may determine the frequency of interactions with other members of the population. The more distant the locations of two agents, the less frequent their interaction. Analogously, agents with similar aspirations are more likely to meet.

Let $\tau$ be a probability measure defined over the Borel-field of $\Lambda \times Y$. Suppose that the marginal distribution of $\tau$ over $\Lambda$ is $\lambda$. Denote the set of all such measures by $\mathcal{D}$. Clearly, $\tau$ is a probability distribution of player characteristics and social ranks $(\alpha, a)$. Hence, for any Borel sets $U \subseteq \Lambda$ and $A \subseteq Y$, value $\tau(U \times A)$ denotes the measure of agents with characteristic $\alpha \in \Lambda$ and $a \in A$.

Given the notation, we define the decision problem faced by a typical agent in the game. The objective of a player is to choose his social status $a \in Y$ that maximizes his expected payoff given by

$$
r(\alpha, a, \tau):=u(|a-y|)+\int_{\Lambda} \int_{Y} v\left(\left|a-a^{\prime}\right|\right) \mathrm{d} \tau\left(a^{\prime} \mid \alpha^{\prime}\right) \mathrm{d} \mu_{\alpha}\left(\alpha^{\prime}\right)
$$


where $\alpha=(x, y)$ and $\tau\left(\cdot \mid \alpha^{\prime}\right)$ is the distribution of actions of other players in the population conditional on $\alpha^{\prime} \in Y$. Therefore, the payoff of an agent is the sum of utilities that he receives from individual interactions with other agents. According to the above definition, the social status of an individual cannot be contingent on the social statuses of other agents, but has to be chosen ex-ante before any interaction occurs. $^{29}$

Formally, since the set of feasible actions is common for all agents and constantly equal to $Y$, Assumption 1(i) is satisfied. By assumption, function $v$ is concave and decreasing. Therefore, Lemma A4 (see the "Appendix") implies that $v\left(\left|a-a^{\prime}\right|\right)$ has increasing differences in $\left(a, a^{\prime}\right)$. It is easy to show that this is sufficient for payoff function $r(\alpha, a, \tau)$ to have increasing differences in $(a, \tau)$ on $Y \times \mathcal{D}$, whenever the set of distributions $\mathcal{D}$ is ordered with respect to the first-order stochastic dominance $\succeq_{P}$. Hence, Assumption 1(ii) is also satisfied. Finally, given that functions $u$ and $v$ are continuous, and assuming that function $\alpha \rightarrow \mu_{\alpha}$ is measurable, Assumption 1(iii) also holds.

By Theorem 1, we conclude there exist a greatest and least distributional equilibria of the game. Furthermore, as function $r(\alpha, a, \tau)$ satisfies the order-continuity on $\mathcal{D}$, which allows for the iterative approximation of the extremal equilibria. Moreover, since agents care about the status of other players as well as their own true identity, the extremal equilibria are trivial only in some special cases. That is, in general it is not the case that, in the greatest (the least) equilibrium of the game, the measure of agents choosing the greatest (the least) possible social status is equal to 1 . Therefore, the approximation methods are useful in determining and computing the distributional equilibria.

An inherent feature of the above example is that agents need to observe the entire distribution of characteristics and actions of other players in order to evaluate their payoffs. In particular, the externality in the game cannot be summarized by an aggregate of actions of players as in Acemoglu and Jensen (2013), as each player's payoff does depend on average distance to their neighbors, rather then distance to the average neighbor. Moreover, since the set of probability distributions is not a lattice, the externality in the game cannot be formulated as a "lattice externality" (see Guesnerie and Jara-Moroni 2011).

Notice that payoffs in this game inherently depend on the joint distribution of player types and actions. Moreover, as the frequency of interactions between individuals depends both on their location and level of aspirations, it is imperative that players have a complete information about the composition of agents in the game. In particular, this implies that the game cannot be reduced to an anonymous large game, in which only the marginal distribution over actions is taken into account by the players. In other words, as characteristics of other players affect consumer payoffs directly, the (semianonymous) equilibrium of the game has to be defined in terms of a joint distribution over characteristics and actions.

\footnotetext{
29 One interpretation of the game is as follows. With probability $\mu_{\alpha}\left(\left\{\alpha^{\prime}\right\}\right)$ player $\alpha$ meets an individual with a characteristic $\alpha^{\prime}$. Then, with probability $\tau\left(\left\{a^{\prime}\right\} \mid \alpha^{\prime}\right)$ player $\alpha^{\prime}$ chooses action $a^{\prime}$. This allows agent $\alpha$ to calculate his expected payoff. In this sense, we can think of this as an interim game. However, we do not analyze ex-post "matching" of agents.
} 
Moreover, note the space of characteristics is not a chain (i.e., is not totally ordered). In fact, it is crucial that the space of characteristics is a subset of the two dimensional real space, as both the location and the true identity of an agent affect his decision. As it was stressed by Akerlof (1997) or Akerlof and Kranton (2000), both the neighborhood effect (the players' location) and the family background effect (the players' true identity) are the two key factors in determining the social interaction and the distribution of equilibrium social distance. However, this means that the space of player characteristics is not a chain. Therefore, the developments of Yang and Qi (2013) cannot be applied in the above framework (see also Balbus et al. 2015c).

\subsection{Linear non-atomic supermodular games}

The previous example is a special case of a larger class of games that we call linear non-atomic supermodular games. Assume a measure space of player characteristics denoted by $(\Lambda, \mathcal{L}, \lambda)$. As in Sect. 2 , let the set of all possible actions be $A$, and the correspondence mapping the characteristics of agents into the set of feasible strategies be denoted by $\tilde{A}$. In addition, we introduce a poset of parameters denoted by $\left(S, \geq_{S}\right)$. In the class of linear non-atomic supermodular games, all the agents in the population interact with each other individually. Therefore, the player's ex-post payoff is a sum of utilities from every separate interaction.

More specifically, suppose the payoff from a single interaction is determined by function $u: \Lambda \times S \times A \times A \rightarrow \mathbb{R}$. That is, an agent with characteristic $\alpha \in \Lambda$, given parameter $s \in S$ and action $a \in \tilde{A}(\alpha)$, yields $u\left(\alpha, s, a, a^{\prime}\right)$ units of utility from an individual interaction with an agent playing $a^{\prime} \in A$. As previously assumed, the frequency of interactions with other players will depend on the trait of a given player. Hence, for any $\alpha \in \Lambda$, there is a non-atomic probability measure $\mu_{\alpha}$ defined over $\mathcal{L}$. Hence, for any measurable group $U \in \mathcal{L}, \mu_{\alpha}(U)$ denotes the probability that an agent with characteristic $\alpha$ will meet an individual with a characteristic belonging to $U$.

Let $\tau \in \mathcal{D}$ be a distribution of characteristics and actions in the population. Given the description, the interim payoff of an agent with $\alpha \in \Lambda$ is

$$
r(\alpha, s, \tau, a):=\int_{\Lambda} \int_{A} u\left(\alpha, s, a, a^{\prime}\right) \mathrm{d} \tau\left(a^{\prime} \mid \alpha^{\prime}\right) \mathrm{d} \mu_{\alpha}\left(\alpha^{\prime}\right) .
$$

Clearly, in order for the payoff to be well defined, we require that function $u(\alpha, s, a, \cdot)$ is $\mathcal{A}$-measurable for any $\alpha, s$, and $a$.

Assume the function $u$ is $\mathcal{L}$-measurable, continuous and supermodular with respect to $a$, and has increasing differences in $\left(a, a^{\prime}\right)$, for any $s \in S$. Moreover, let $\mu_{\alpha}$ be measurable as a function of $\alpha$. Given Theorem 1, any such linear non-atomic supermodular game has a greatest and a least distributional equilibrium, which can be approximated using our iterative method.

Assume for any $\alpha \in \Lambda$ and $a^{\prime \prime} \geq a$ in $\tilde{A}(\alpha)$, the family of functions $\left\{\Delta_{\alpha}\left(\cdot, a^{\prime}\right)\right\}_{a^{\prime} \in A}$, where $\Delta_{\alpha}\left(s, a^{\prime}\right):=u\left(\alpha, s, a^{\prime \prime}, a^{\prime}\right)-u\left(\alpha, s, a, a^{\prime}\right)$, obey the signed-ratio monotonicity (see Quah and Strulovici 2012). Then, the function $r(\alpha, s, \tau, a)$ has single crossing differences in $(a, s)$ on $A \times S$. By Corollary 1 , the greatest and the least distributional equilibrium of the game increases with respect to the deep parameter $s$. 
The key feature of the above class of games is that the payoff function is linear with respect to measure $\tau$. Therefore, it is weakly continuous on the space of probability measures. This implies that the additional order-continuity assumption imposed prior to Theorem 1(iii) is always satisfied. Hence, no additional assumptions have to be imposed in order for the result to hold.

\subsection{Large stopping games}

We next turn to an optimal stopping time example. Suppose that a continuum of agents are deciding how long should each one participate in an investment project that lasts at most $T$ periods. Each period $t$, each agent takes part in the investment, from which she receives a profit of $\pi(t, m)$, where $m$ is the measure of agents participating in the project at time $t$. We assume $\pi(t, m)$ may take both positive and negative values; however, it is increasing in $m$. In other words, the more agents participate in the project, the higher are the profits (or, lower are the losses) to every individual agent. Finally, whenever the agent is not participating at the project, her payoff is equal to zero.

In the following analysis, we concentrate solely on the case where time is discrete. Define the set of time indices by $\{1,2, \ldots, T\}$. Suppose the time at which the agent joins the project is determined exogenously and that it defines the characteristic of an agent. Hence, $\alpha \in \Lambda$, where $\Lambda:=\{1,2, \ldots, T\}$. The distribution of characteristics across agents is determined by some measure $\lambda$ over $\Lambda$. Hence, $\lambda(\alpha)$ is the measure of agents that join the project at time $\alpha$. Endow $\Lambda$ with a natural order.

Since the time at which agents join the investment is given exogenously, they can only decide when to leave the investment. Assume that agents can leave the project only once (i.e., just like in a standard optimal stopping game). Given this, an action of a player is equivalent to a time index at which the agent decides to leave the investment. Using our notation, the set of all possible actions $A$ is equivalent to the set of time indices $\Lambda .{ }^{30}$ Moreover, the correspondence mapping agents characteristics into the set of feasible actions is defined by $\tilde{A}(\alpha):=\{\alpha, \ldots, T\}$.

Assume the distribution of characteristics and actions for the population is given by $\tau \in \mathcal{D}$. Therefore, for any $(\alpha, a) \in \Lambda \times A, \tau(\{(\alpha, a)\})$ is the measure of agents joining the investment at time $\alpha$ and leaving at time $a$. Define function $F: \mathcal{D} \times \Lambda \rightarrow[0,1]$ as

$$
F(\tau, t):=\tau(\{(\alpha, a): \alpha \leq t \leq a\})
$$

In other words, $F(\tau, t)$ is a measure of agents participating in the project at time $t$. Note that function $F(\tau, \cdot)$ is not a probability distribution nor a cumulative distribution. Clearly, the sum of its values might not be equal to one and it need not be monotone.

Given the notation, we define the payoff of an agent $\alpha \in \Lambda$ by

$$
r(\alpha, a, \tau):=\sum_{t=\alpha}^{a} \pi(t, F(\tau, t)),
$$

\footnotetext{
30 We shall differentiate the notation of these two sets in order to avoid confusion.
} 
The objective of every agent is to maximize $r(\alpha, \tau, a)$ with respect to $a \in \tilde{A}(\alpha)$.

To show the above game obeys Assumption 1, we first show that function $F(\tau, \cdot)$ is pointwise increasing as the measures $\tau \in \mathcal{D}$ shifts upward with respect to the first-order stochastic dominance. Consider any $\tau^{\prime}$ and $\tau$ in $\mathcal{D}$ such that $\tau^{\prime} \succeq_{P} \tau$. By definition of the first-order stochastic dominance, this implies that for any $(\alpha, a) \in \Lambda \times \Lambda$, we have

$$
\tau^{\prime}\left(\left\{\left(\alpha^{\prime}, a^{\prime}\right):\left(\alpha^{\prime}, a^{\prime}\right) \leq(\alpha, a)\right\}\right) \leq \tau\left(\left\{\left(\alpha^{\prime}, a^{\prime}\right):\left(\alpha^{\prime}, a^{\prime}\right) \leq(\alpha, a)\right\}\right) .
$$

Since $\tau^{\prime}$ and $\tau$ belong to $\mathcal{D}$, their marginals over the space of characteristics $\Lambda$ are the same. Therefore, $\tau^{\prime}\left(\left\{\left(\alpha^{\prime}, a^{\prime}\right): a^{\prime} \leq a\right\}\right) \leq \tau\left(\left\{\left(\alpha^{\prime}, a^{\prime}\right): a^{\prime} \leq a\right\}\right)$. Then,

$$
F(\tau, t):=\tau(\{(\alpha, a): \alpha \leq t \leq a\}) \leq \tau^{\prime}(\{(\alpha, a): \alpha \leq t \leq a\})=: F\left(\tau^{\prime}, t\right),
$$

for any $t \in \Lambda$. The above property is sufficient for the payoff $r$ to have increasing differences in $(a, \tau)$ on $A \times \mathcal{D}$. Clearly, since function $\pi(t, \cdot)$ is increasing,

$$
\begin{aligned}
r\left(\alpha, a^{\prime}, \tau\right)-r(\alpha, a, \tau) & =\sum_{t=a}^{a^{\prime}} \pi(t, F(\tau, t)) \\
& \leq \sum_{t=a}^{a^{\prime}} \pi\left(t, F\left(\tau^{\prime}, t\right)\right)=r\left(\alpha, \tau^{\prime}, a^{\prime}\right)-r\left(\alpha, \tau^{\prime}, a\right),
\end{aligned}
$$

for any $\alpha \in \Lambda$ and $a^{\prime} \geq a$ in $A$. To complete the argument, note as the space of characteristics and actions is finite, the payoff function is trivially continuous on $\Lambda \times A$. Therefore, Assumption 1 is satisfied. Moreover, once we assume that function $\pi(t, \cdot)$ is continuous for any $t \in \Lambda$, the payoff function is order-continuous with respect to $\tau$. By Theorem 1, we conclude the game has a greatest and a least distributional equilibrium which can be approximated using our iterative methods.

Finally, we are able to determine the comparative statics of the extremal equilibria. Assume each period, the payoff function is parameterized by a deep parameter $s$ belonging to a poset $\left(S, \geq_{S}\right)$. Hence, each period the agent that participates in the investment receives $\pi(t, s, m)$, where $m$ is the measure of players taking part in the project at the given time. Suppose $\pi(t, \cdot, m)$ is an increasing function for any $t \in \Lambda$ and $m \in[0,1]$. Clearly, for any $\alpha$ and $\tau$,

$$
r(\alpha, s, a, \tau):=\sum_{t=\alpha}^{a} \pi(t, s, F(\tau, t)),
$$

has increasing differences in $(a, s)$ on $A \times S$. By Corollary 1 , this is sufficient to conclude that the extremal equilibria are increasing functions of $s$.

As in the model of social distance, in order to define payoffs in the above game, it is imperative to specify the joint distribution over player characteristics and actions. Clearly, given that the number of agents currently present in the market is determined by the number of agents who have already arrived and those who have not yet left, both 
the arrival time (i.e., the characteristic) and the stopping time (i.e., the action) of other players have to be taken into account while evaluating the payoff of each individual.

\subsection{Keeping up with the Joneses}

We finish with an application of our results to large economies with peer effects. Consider an economy consisting of a continuum of consumers. Every consumer in the economy is characterized by their initial wealth $m \geq 0$, and a number $i \in[0,1]$. In our framework, the number $i$ will correspond to the relative social position to which the agent refers to when choosing her consumption level. We formalize the notion in the remainder of this subsection.

Let the set off all possible values of wealth $m$ be a compact subset $M$ of $\mathbb{R}_{+}$, where $\bigvee M:=\bar{m}$. Let $\Lambda:=M \times[0,1]$, and $\alpha=(m, i)$ be one of its elements. The distribution of characteristics is determined by a measure $\lambda$ over the product Borelfield $\mathcal{L}$ of $\lambda$.

There are two markets in the economy: the consumption good market and the labor market. Every agent is endowed with $m$ units of the initial wealth, expressed in units of the consumption good, and one unit of time that can be devoted either to labor or leisure. Given the normalized price of consumption $p=1$ and wage $w>0$, the budget set is

$$
B(m, w)=\left\{(a, n) \in \mathbb{R}_{+} \times[0,1]: m+w \geq a+w n\right\},
$$

where by $(a, n)$ we denote a pair of consumption $a$ and leisure $n$. Note that the set of feasible consumption levels in our framework is given by $A=[0, \bar{m}+w]$.

Apart from the consumption and leisure, every agent takes into account the relative level of her consumption to other agents in the economy. Number $i \in[0,1]$, characterizing the consumer, denotes the quantile of the distribution of consumption in the population that the agent is treating as a reference point when choosing her consumption. In other words, the higher is the agent's consumption above the $i$ th quantile of the distribution, the better. This feature of the model incorporates the keep up with the Joneses effect, but in a heterogenous manner. Thus, each agent is characterized by a different $i$.

Assume that the distribution of characteristics and consumption in the economy is defined by a probability measure $\tau$ over $\Lambda \times A$. Let

$$
q(\tau, i):=\min \left\{a^{\prime} \in A: \tau\left(\left\{(\alpha, a) \in \Lambda \times A: a \leq a^{\prime}\right\}\right) \geq i\right\} .
$$

Hence, $q(\tau, i)$ is the $i$ th quantile of the distribution of consumption, given $\tau$. This implies that the game cannot be reduced to aggregative one.

Every consumer is endowed with a pair of utilities $u: \mathbb{R}_{+} \times[0,1] \rightarrow \mathbb{R}_{+}$and $v: \mathbb{R} \rightarrow \mathbb{R}$. We assume both functions are continuous, increasing, and concave. Given the distribution of consumption $\tau$, the objective of every agent is to solve

$$
\max _{(a, n) \in B(m, w)} u(a, n)+v(a-q(\tau, i)) .
$$


When choosing her consumption and leisure, agent takes into account their direct effect summarized by function $u$, as well as the utility yielded from the relative consumption.

Note that in the above framework, we model the social status differently than in the example discussed in Sect. 3.1. Since agents' utility depends positively on the difference between their consumption and a certain quantile of the distribution of consumption, the social status is defined relatively to the mass of agents that consume less than the given player. We consider this to be a good approximation of the consumer choice, when social class concerns are taken into consideration-agents want their consumption to dominate the consumption of a certain fraction of the population.

Given monotonicity of $u$ and $v$, the budget constraint is always binding. Hence, we have $n=(m+w-a) / w$, for any optimal solution $(a, n)$. This allows us to reduce the number of variables in the consumer optimization problem. Given the condition, the payoff of the consumer can be expressed by

$$
r(\alpha, a, \tau):=u(a,(m+w-a) / w)+v(a-q(\tau, i)),
$$

where $\alpha=(m, i)$, while the set of feasible strategies is $\tilde{A}(\alpha)=[0,(w+m) / w]$. Hence, given $\tau$, the objective is to maximize $r(\alpha, a, \tau)$ with respect to $a \in \tilde{A}(\alpha)$.

In order to apply our main result to the above example, we need to show it satisfies the conditions of Assumption 1. Clearly, set $A$ is a lattice, while correspondence $\tilde{A}$ is continuous and complete lattice valued. By assumptions imposed on functions $u$ and $v$, function $r$ is continuous in $a$ and measurable with respect to the characteristic $\alpha$. Therefore, it suffices to show that is has single crossing differences in $(a, \tau)$.

First, note that the quantile function $q(\cdot, i)$ is increasing on $\left(\mathcal{D}, \succeq_{P}\right)$, for any $i$. Take any $a^{\prime} \geq a$ in $\tilde{A}(\alpha)$ and $\tau^{\prime} \succeq_{P} \tau$ in $\mathcal{D}$. Then,

$$
\begin{aligned}
& r\left(\alpha, a^{\prime}, \tau\right)-r(\alpha, a, \tau)=v\left(a^{\prime}-q(\tau, i)\right)-v\left(a^{\prime}-q(\tau, i)\right) \\
& \quad \leq v\left(a^{\prime}-q\left(\tau^{\prime}, i\right)\right)-v\left(a^{\prime}-q\left(\tau^{\prime}, i\right)\right)=r\left(\alpha, a^{\prime}, \tau^{\prime}\right)-r\left(\alpha, a, \tau^{\prime}\right),
\end{aligned}
$$

where the inequality is implied by monotonicity of $q(\cdot, i)$ and Lemma A3 (see the "Appendix"). Hence, function $r(\alpha, \tau, a)$ has increasing differences in $(a, \tau)$. Therefore, the above example satisfies the conditions stated in Assumption 1 and our results follow.

The above observation implies that the game presented above admits a greatest and a least distributional equilibrium. Moreover, our results allow to determine some equilibrium comparative statics. First of all, as function $q(\tau, \cdot)$ is increasing in $i$, it is easy to show that objective $r$ function has increasing differences in $(a, i)$. This implies that the greatest and the least distributional equilibria shift in the first-order stochastic sense as the characteristic $i$ increases for all agents. Hence, the marginal distribution over equilibrium consumption increases stochastically with individual aspirations $i$.

Analogously, whenever we assume consumption is a normal good, we can show that equilibrium consumption increases stochastically with respect to the profile of 
the initial wealth $m .^{31}$ Therefore, the higher is the initial wealth $m$ of each agent, the greater is the marginal distribution of equilibrium consumption in the economy.

Our approach to status and conspicuous consumption differs in several ways from the one presented in Hopkins and Kornienko (2004, 2009). First of all, our notion of status is defined as a level of consumption chosen by the individual relatively to a particular quantile of the distribution of consumption in the economy. Therefore, value $q(\tau, i)$ determines a benchmark, expressed in units of consumption, that the consumer is using to evaluate her status. In contrast, Hopkins and Kornienko (2004) define status a measure of agents whose consumption is lower than the consumer's. In particular, unlike in our case, the above notion implies that the steeper is the distribution of consumption in the economy, the higher is the marginal utility the agent receives from attaining higher status. In our case, changes in utility from status depend only on the benchmark quantile $q(\tau, i)$. See also Gavrel and Rebière (2017).

Our specification of status allows us to analyze problems in which heterogeneity in aspiration levels plays a significant role. In contrast to Hopkins and Kornienko (2004), every agent in our economy is characterized both by the initial wealth $m$ as well as the parameter $i$. Despite the heterogeneity, employing the tools introduced in the preceding sections allows us to determine existence of extreme equilibria and some comparative statics of the equilibrium outcome. On the other hand, the analysis of Hopkins and Kornienko (2004) requires for agents to be symmetric (up to the level of initial wealth). Clearly, their approach has many advantages. In particular, they are able to determine existence of a unique equilibrium of the game and provide some important comparative statics results. However, this comes at the cost of reducing heterogeneity (and semi-anonymity) in the economy.

Acknowledgements We thank Rabah Amir, Bob Becker, Ed Green, Martin Kaae Jensen, Ali Khan, Bob Lucas, Alejandro Manelli, Ed Prescott, Xavier Vives, Jan Werner, and Nicholas Yannelis for helpful discussions on various issues related to this paper. We also thank the Editor and two anonymous Referees of this journal for their comments on the earlier drafts of this paper. The Project was financed by NCN Grant No. UMO-2012/07/D/HS4/01393. Reffett thanks the Dean's Award in Excellent Summer Grant Program for its generous support of this research.

Open Access This article is distributed under the terms of the Creative Commons Attribution 4.0 International License (http://creativecommons.org/licenses/by/4.0/), which permits unrestricted use, distribution, and reproduction in any medium, provided you give appropriate credit to the original author(s) and the source, provide a link to the Creative Commons license, and indicate if changes were made.

\section{A Appendix}

In the following section, we present several auxiliary results used in the arguments supporting our main theorems. First, we present a theorem by Markowsky (1976). See Theorem 9 of his paper for the original reference.

Theorem A1 (Markowsky's fixed-point theorem) Let $F: X \rightarrow X$ be increasing, and $X$ be a chain complete poset. Then, the set of fixed points of $F$ is a chain complete

\footnotetext{
31 Following Quah (2007), it suffices to assume that function $u$ is concave and supermodular.
} 
poset. Moreover, $\bar{x}:=\bigvee\{x: x \leq F(x)\}$ and $\underline{x}:=\bigwedge\{x: x \geq F(x)\}$ constitute a greatest and a least fixed point of $F$ respectively.

In many cases, we are referring to a constructive version of Markowsky's Theorem. The Tarski-Kantorovich fixed-point theorem is one such theorem. We now provide a generalization of the Tarski-Kantorovich result that additionally provides a fixedpoint comparative statics results in the spirit of Veinott (1992) version of Tarski's theorem. See also Balbus et al. (2015b), where it was originally stated and proved. For a monotone sequence $\left\{x_{n}\right\}_{n=0}^{\infty}$, let

$$
\bigvee x_{n}:=\sup _{n \in \mathbb{N}} x_{n} \text { and } \bigwedge x_{n}:=\inf _{n \in \mathbb{N}} x_{n}
$$

By $F^{n}(x)$, we denote the $n$th orbit (or iteration) of function $F$ from point $x \in X$. That is, $F^{n}(x):=F \circ F \circ \cdots \circ F(x)$.

Definition 1 Function $F: X \rightarrow X$ is monotonically sup-preserving (monotonically inf-preserving) if, for any monotone sequence $\left\{x_{n}\right\}_{n=0}^{\infty}$, we have $F\left(\bigvee x_{n}\right)=\bigvee F\left(x_{n}\right)$ (respectively $\left.F\left(\bigwedge x_{n}\right)=\bigwedge F\left(x_{n}\right)\right) . F$ is monotonically sup/inf-preserving if and only if it is both monotonically sup- and inf-preserving.

It is worth mentioning a monotonically sup- or inf-preserving function is necessarily increasing. The Tarski-Kantorovich Theorem (see Theorem 4.2 in Dugundji and Granas 1982) states the following.

Theorem A2 (Tarski-Kantorovitch fixed-point theorem) Let $X$ be a countably chain complete poset with the greatest and the least element denoted by $\bar{x}$ and $\underline{x}$ respectively. Let $\Phi$ denote the set of fixed points of function $F: X \rightarrow X$. Then,

(i) if $F$ is monotonically inf-preserving, $\bigwedge F^{n}(\bar{x})$ is a greatest fixed point of $F$;

(ii) if $F$ is monotonically sup-preserving, $\bigvee F^{n}(\underline{x})$ is a least fixed point of $F$.

Next, we present two new theorems that can be developed using the TarskiKantorovitch fixed-point theorem. First, we characterize the set of fixed point of function $F$. The second result provides the monotone comparative statics of the set.

Theorem A3 Let X be a countably chain complete poset, $F: X \rightarrow X$ a monotonically sup/inf-preserving function, and $\Phi$ is the set of fixed points of $F$. Then, set $\Phi$ is a nonempty countably chain complete poset with a greatest and a least element denoted by

$$
\bar{\phi}:=\bigvee\{x: F(x) \geq x\} \quad \text { and } \quad \underline{\phi}:=\bigwedge\{x: F(x) \leq x\}
$$

Theorem A4 Let $X$ be a countably chain complete poset with greatest and least element, $T$ be a poset, and $F: X \times T \rightarrow X$ be an increasing function such that $F(\cdot, t)$ is monotonically inf-preserving (monotonically sup-preserving) on $X$, for all $t \in T$. Moreover, denote the greatest (the least) fixed point of $F(\cdot, t)$ by $\bar{\phi}(t)(\underline{\phi}(t))$, for some $t \in T$. Then, function $t \rightarrow \bar{\phi}(t)(t \rightarrow \underline{\phi}(t))$ is increasing. 
See Balbus et al. (2015b) for the proofs of Theorems A3 and A4. Next, we prove a space of regular measures defined over a compact support and endowed with the first-order stochastic dominance order is a chain complete poset. Suppose $(X, \geq)$ is a partially ordered set, where $X$ is a compact space endowed with Hausdorff topology $\mathcal{X}$ and a Borel $\sigma$-field $\mathcal{B}$. By $C(X)$ we denote the set of continuous, real-valued functions on $X$. Let $M(X)$ be the set of isotone, measurable, and bounded real-valued functions over $X$. We say a measure $\mu$ first-order stochastically dominates a measure $v$, denoted $\mu \succeq_{P} v$, whenever

$$
\int_{X} f \mathrm{~d} \mu \geq \int_{X} f \mathrm{~d} \nu, \text { for all } f \in M(X) .
$$

Let $\mathcal{R}$ be a set of regular measures over $X$.

Lemma A1 If $M(X)$ separates the points of $X$, then $\left(\mathcal{R}, \succeq_{P}\right)$ is an ordered space.

Proof Clearly $\succeq_{P}$ is reflexive and transitive. We now need to show it is antisymmetric. Assume $\mu \succeq_{P} v$ and $v \succeq_{P} \mu$. Then.

$$
\int_{X} f \mathrm{~d} \mu=\int_{X} f \mathrm{~d} \nu, \text { for all } f \in M(X) .
$$

Clearly, set $M(X)-M(X)$ is a Riesz subspace of $C(X)$ and contains a constant function. ${ }^{32}$ Therefore, by the Stone-Weierstrass Theorem (see Aliprantis and Border 2006, Theorem 9.12) $M(X)-M(X)$ is uniformly dense on $C(X)$. In particular, condition (3) is satisfied for all $f \in C(X)$.

Take any two closed subsets $F$ and $F^{\prime}$ of $X$ such that $F \cap F^{\prime}=\emptyset$, while $\mu\left(F \cup F^{\prime}\right)$ and $\nu\left(F \cup F^{\prime}\right)$ are arbitrarily close to 1 . Clearly, since $X$ is compact and Hausdorff, any closed set is compact. Since the two measures are regular (hence, tight) such subsets exist. Let an arbitrary $\varepsilon>0$ be such that $\mu\left(F \cup F^{\prime}\right)>1-\varepsilon$ and $\nu\left(F \cup F^{\prime}\right)>1-\varepsilon$. As $X$ is compact and Hausdorff, by Urysohn's Lemma (see Aliprantis and Border 2006, Lemma 2.46), it is possible to construct a continuous function $f: X \rightarrow[0,1]$ that satisfies $f(x)=1$, for $x \in F$, and $f(x)=0$, for $x \in F^{\prime}$. Since $\int_{X} f \mathrm{~d} \mu=\int_{X} f \mathrm{~d} \nu$, we obtain

$$
\begin{aligned}
v(F) & \leq v(F)+\int_{F^{c} \cap F^{\prime c}} f \mathrm{~d} v=\int_{X} f \mathrm{~d} v \\
& =\int_{X} f \mathrm{~d} \mu=\mu(F)+\int_{F^{c} \cap F^{\prime c}} f \mathrm{~d} \mu \leq \mu(F)+\varepsilon .
\end{aligned}
$$

Analogously, we can show that $\mu(F) \leq v(F)+\varepsilon$. Given that $\varepsilon$ is arbitrary, this implies that $\mu(F)=v(F)$, for any closed (hence, compact) $F \subseteq X$. Since the measures are tight, this implies that $\mu=v$.

Given the above preliminary result, we may proceed with our characterization of the ordered space of regular measures.

\footnotetext{
32 See Aliprantis and Border (2006, p. 646, footnote 5).
} 
Proposition A1 $\left(\mathcal{R}, \succeq_{P}\right)$ is a chain complete poset.

Proof of Proposition Al Let $\left\{\tau_{t}\right\}$ be a decreasing chain. Recall $M(X)$ is the set of isotone, measurable, and bounded real-valued functions over $X$. Define $T: M(X) \rightarrow$ $\mathbb{R}$,

$$
T(f):=\inf _{t}\left\{\int_{X} f(s) \mathrm{d} \tau_{t}(s)\right\},
$$

and for antitone functions $f \in-M(X)$

$$
T(f)=-\inf _{t}\left\{\int_{X}-f(s) \mathrm{d} \tau_{t}(s)\right\}=\sup _{t}\left\{\int_{X} f(s) \mathrm{d} \tau_{t}(s)\right\} .
$$

Note that, $T$ is a functional on $M(X)$ that preserves addition and multiplication by a positive scalar. It can be extended to the vector subspace $M(X)-M(X)$ as follows. If $f=g-h$, for $g, h \in M(X)$, then $T(f)=T(g)-T(h)$, which is well defined. Indeed, if $f \in M(X)-M(X)$, then $f=g-(g-f)$, for $g \in M(X)$ and $f-g \in M(X)$. Hence,

$$
\inf _{t} \int_{X} g(x) \mathrm{d} \tau_{t}(x)=\lim _{t} \int_{X} g(x) \mathrm{d} \tau_{t}(x),
$$

as well as

$$
\inf _{t} \int_{X} g(x) \tau_{t}(\mathrm{~d} x)=\lim _{t} \int_{X} f(x)-g(x) \tau_{t}(\mathrm{~d} x) .
$$

Since $T$ is isotone,

$$
|T(f)| \leq\|f\|_{\infty},
$$

while the inequality may hold as $T(1)=1$. By the Banach Extension Theorem, there exists an extension $\hat{T}$ of $T$ for all elements in $C(X)$ that satisfy (4). By the Riesz Representation Theorem (see Aliprantis and Border 2006, Theorem 14.12) there is a unique regular measure $\underline{\tau}$ such that

$$
\hat{T}(f)=\int_{X} f(s) \mathrm{d} \underline{\tau}(s),
$$

for all $f \in M(X)$. Moreover, by condition (4), it is a probability measure. Clearly, $\underline{\tau}$ is a lower bound of the chain $\left\{\tau_{t}\right\}$. We need to show it is the greatest lower bound in $\mathcal{R}$.

We prove our claim by contradiction. Suppose there is another measure $\tau_{0}$ which is a lower bound of $\left\{\tau_{t}\right\}$, but it is not dominated by $\tau$. Then, there exists a function $f \in M(X)$ such that $\int_{X} f \mathrm{~d} \tau_{0}>\int_{X} f \mathrm{~d} \underline{\tau}$. As $\tau_{0}$ is a lower bound of $\left\{\tau_{t}\right\}$, we have

$$
T(f)=\inf _{t}\left\{\int_{X} f(s) \mathrm{d} \tau_{t}(s)\right\} \geq \int_{X} f \mathrm{~d} \tau_{0}>\int_{X} f \mathrm{~d} \underline{\tau}=T(f),
$$

which yields a contradiction. Hence, $\underline{\tau}$ is the greatest lower bound of $\left\{\tau_{t}\right\}$. Similarly, we can prove the thesis for increasing chains.

Next, we present an auxiliary result that is used in the proof of Lemma 1. 
Lemma $\mathrm{A} 2$ Let $X$ and $Y$ be Hausdorff topological spaces endowed with $\sigma$-fields $\mathcal{B}_{X}$ and $\mathcal{B}_{Y}$ respectively. Let $\mu$ be a finite measure on $\mathcal{B}_{X} \otimes \mathcal{B}_{Y}$. Whenever the marginals of $\mu$ on $X$ and $Y$ are regular measures, then $\mu$ is a regular measure.

Proof By Theorem 12.4 in Aliprantis and Border (2006), it is sufficient to show that measure $\mu$ is tight. Define

$$
\mathcal{E}:=\left\{V \in \mathcal{B}_{X} \otimes \mathcal{B}_{Y}: \mu(V)=\sup \{\mu(K): K \subseteq V, K \text { is compact }\}\right\}
$$

By the standard argument (see the proof of Theorem 12.5 in Aliprantis and Border 2006), $\mathcal{E}$ is a $\sigma$-field. We need to show that $\mathcal{E}$ includes sets of the form $U_{x} \times U_{y}$, with $U_{x} \in \mathcal{B}_{X}$ and $U_{y} \in \mathcal{B}_{Y}$. Since the marginals of $\mu$ are tight, hence for given $\varepsilon>0$, there are compact sets $K_{x} \subset U_{x}$ and $K_{y} \subset Y_{y}$ such that $\mu_{x}\left(U_{x} \backslash K_{x}\right)<\varepsilon / 2$ and $\mu_{y}\left(U_{y} \backslash K_{y}\right)<\varepsilon / 2$, where $\mu_{x}$ and $\mu_{y}$ denote the marginals of $\mu$ on $X$ and $Y$, respectively. Then,

$$
\mu\left(\left(U_{x} \times U_{y}\right) \backslash\left(K_{x} \times K_{y}\right)\right) \leq \mu\left(\left(U_{x} \backslash K_{x}\right) \times Y\right)+\mu\left(X \times\left(Y \backslash K_{y}\right)\right)<\varepsilon .
$$

Hence, $U_{x} \times U_{y} \in \mathcal{E}$.

Finally, we present one result applied in Sect. 3.1.

Lemma A3 Let $f: X \rightarrow \mathbb{R}$ be a concave function over a convex subset $X \subseteq \mathbb{R}$. Define $Y:=\left\{(x, s) \in \mathbb{R}^{2}:(x-s) \in X\right\}$. Then, function $g: Y \rightarrow \mathbb{R}$, where $g(x, s):=$ $f(x-s)$, has increasing differences in $(x, s)$ on $X \times X$.

Proof of Lemma A3 Since $f$ is concave, for any $x^{\prime} \geq x$, and $s^{\prime} \geq s$, we have

$$
\frac{f\left(x^{\prime}-s^{\prime}\right)-f\left(x-s^{\prime}\right)}{\left(x^{\prime}-s^{\prime}\right)-\left(x-s^{\prime}\right)} \geq \frac{f\left(x^{\prime}-s\right)-h(x-s)}{\left(x^{\prime}-s\right)-(x-s)},
$$

which implies that $f\left(x^{\prime}-s^{\prime}\right)-f\left(x-s^{\prime}\right) \geq f\left(x^{\prime}-s\right)-f(x-s)$.

Lemma A4 Let $f: X \rightarrow \mathbb{R}$, where $X \subseteq \mathbb{R}_{+}$is convex, be a decreasing and concave function. Let $Y:=\left\{(x, s) \in \mathbb{R}^{2}:|x-s| \in X\right\}$. Then, function $g: Y \rightarrow \mathbb{R}$, defined by $g(x, s):=f(|x-s|)$ has increasing differences in $(x, s)$.

Proof of Lemma A4 First, we prove function $h: X \rightarrow \mathbb{R}, h(x):=f(|x|)$, is concave. Take any $x^{\prime}, x \in X$ and $\alpha \in[0,1]$. Then

$$
f\left(\left|\alpha x+(1-\alpha) x^{\prime}\right|\right) \geq f\left(\alpha|x|+(1-\alpha)\left|x^{\prime}\right|\right) \geq \alpha f(|x|)+(1-\alpha) f\left(\left|x^{\prime}\right|\right),
$$

where the first inequality is implied by the triangle inequality and monotonicity of $f$, while the second follows from concavity of $f$. The rest is implied by Lemma A3. 


\section{References}

Acemoglu, D., Jensen, M.K.: Robust comparative statics in large static games. In: IEEE Proceedings on Decision and Control, pp. 3133-3139 (2010)

Acemoglu, D., Jensen, M.K.: Aggregate comparative statics. Games Econ. Behav. 81, 27-49 (2013)

Akerlof, G.: Social distance and social decisions. Econometrica 65(5), 1005-1028 (1997)

Akerlof, G., Kranton, R.: Economics and identity. Q. J. Econ. 115(3), 715-753 (2000)

Aliprantis CD, Border KC (2006) Infinite Dimentional Analysis. A Hitchhiker's Guide, 3rd edn. Springer, Heilbelberg

Balbus, Ł., Dziewulski, P., Reffett, K., Woźny, Ł.: Differential information in large games with strategic complementarities. Econ. Theory 59(1), 201-243 (2015a). doi:10.1007/s00199-014-0827-x

Balbus, Ł., Reffett, K., Woźny, Ł.: Computing time-consistent Markov policies for quasi-hyperbolic consumers under uncertainty. Int. J. Game Theory 44(1), 83-112 (2015b)

Balbus, Ł., Reffett, K., Woźny, Ł.: Monotone equilibria in nonatomic supermodular games. A comment. Games Econ. Behav. 94, 182-187 (2015c)

Bilancini, E., Boncinelli, L.: Strict Nash equilibria in non-atomic games with strict single crossing in players (or types) and actions. Econ. Theory Bull. 4, 95-109 (2016)

Carmona, G., Podczek, K.: Existence of Nash equilibrium in games with a measure space of players and discontinuous payoff functions. J. Econ. Theory 152, 130-178 (2014)

Davis, A.: A characterization of complete lattices. Pac. J. Math. 5, 311-319 (1955)

Dugundji, J., Granas, A.: Fixed Point Theory. PWN Polish Scientific Publishers, Warsaw (1982)

Gavrel, F., Rebière, T.: On the equilibrium and welfare consequences of getting ahead of the Smiths. J. Public Econ. Theory. (2017). doi:10.1111/jpet.12261

Guesnerie, R., Jara-Moroni, P.: Expectational coordination in simple economic contexts. Econ. Theory 47(2), 205-246 (2011). doi:10.1007/s00199-010-0556-8

Heikkilä, S., Reffett, K.: Fixed point theorems and their applications to theory of Nash equilibria. Nonlinear Anal. 64, 1415-1436 (2006)

Hopkins, E., Kornienko, T.: Running to keep in the same place: Consumer choice as a game of status. Am. Econ. Rev. 94(4), 1085-1107 (2004)

Hopkins, E., Kornienko, T.: Status, affluence, and inequality: rank-based comparisons in games of status. Games Econ. Behav. 67(2), 552-568 (2009)

Kamae, T., Krengel, U., O’Brien, G.L.: Stochastic inequalities on partially ordered spaces. Ann. Prob. 5(6), 899-912 (1977)

Khan, M.: Equilibrium points of nonatomic games over a banach space. Trans. Am. Math. Soc. 293, 737-749 (1986)

Khan, M.A., Rath, K.P., Sun, Y., Yu, H.: Large games with a bio-social typology. J. Econ. Theory 148(3), 1122-1149 (2013a)

Khan, M.A., Rath, K.P., Yu, H., Zhang, Y.: Large distributional games with traits. Econ. Lett. 118(3), 502-505 (2013b)

LiCalzi M, Veinott AF (1992) Subextremal Functions and Lattice Programming. Standford University

Markowsky, G.: Chain-complete posets and directed sets with applications. Algebra Univ. 6, 53-68 (1976)

Mas-Colell, A.: On a theorem of Schmeidler. J. Math. Econ. 13(3), 201-206 (1984)

Milgrom, P., Roberts, J.: Rationalizability, learning and equilibrium in games with strategic complementarites. Econometrica 58(6), 1255-1277 (1990)

Milgrom, P., Shannon, C.: Monotone comparative statics. Econometrica 62(1), 157-180 (1994)

Prokopovych, P., Yannelis, N.C.: On strategic complementarities in discontinuous games with totally ordered strategies. J. Math. Econ. 70, 147-153 (2017)

Quah, J.K.H.: The comparative statics of constrained optimization problems. Econometrica 75(2), 401-431 (2007)

Quah, J.K., Strulovici, B.: Aggregating the single crossing property. Econometrica 80(5), 2333-2348 (2012)

Rath, K.P.: Existence and upper hemicontinuity of equilibrium distributions of anonymous games with discontinuous payoffs. J. Math. Econ. 26(3), 305-324 (1996)

Schmeidler, D.: Equilibrium points of nonatomic games. J. Stat. Phys. 17(4), 295-300 (1973)

Shannon C (1990) An ordinal theory of games with strategic complementarities. Working paper, Standford University

Sun, X., Zhang, Y.: Pure-strategy Nash equilibria in nonatomic games with infinite-dimensional action spaces. Econ. Theory 58(1), 161-182 (2015). doi:10.1007/s00199-013-0795-6 
Tarski, A.: A lattice-theoretical fixpoint theorem and its applications. Pac. J. Math. 5, 285-309 (1955)

Topkis, D.M.: Equilibrium points in nonzero-sum n-person submodular games. SIAM J. Control Optim. 17, 773-787 (1979)

Topkis, D.M.: Supermodularity and Complementarity. Princeton University Press, Princeton, Frontiers of Economic Research (1998)

Veinott AF (1992) Lattice programming: qualitative optimization and equilibria. MS Standford

Vives, X.: Nash equilibrium with strategic complementarites. J. Math. Econ. 19, 305-321 (1990)

Yang, J., Qi, X.: The nonatomic supermodular game. Games Econ. Behav. 82, 609-620 (2013)

Zhou, L.: The set of Nash equilibria of a supermodular game is a complete lattice. Games Econ. Behav. 7, 295-300 (1994) 